\title{
Encephalopathy Associated with Ifosfamide Use in the Treatment of Patient with Diffuse Large B Cell Lymphoma
}

\author{
Noor Sameh Darwich ${ }^{1, *}$, and Ibrahim Mustafa ${ }^{2}$ \\ ${ }^{1}$ Miami valley Hospital, Dayton, $\mathrm{OH}, \mathrm{USA}$ \\ ${ }^{2}$ Department of Neurology and Neuroscience, Wright state University, Miami Valley Hospital, Dayton, $\mathrm{OH}, \mathrm{USA}$
}

*Corresponding author: Noor Sameh Darwich, Miami valley Hospital, One Wyoming Street, Dayton, OH 45409, USA, Email: nsdarwich@gmail.com

Received: 03 Apr, 2021 | Accepted: 07 May, 2021 | Published: 15 May, 2021

Citation: Darwich NS, Mustafa I (2021) Encephalopathy Associated with Ifosfamide Use in the Treatment of Patient with Diffuse Large B Cell Lymphoma. J Neurol Neurobiol 7(1): dx.doi.org/10.16966/2379-7150.174

Copyright: (C) 2021 Darwich NS, et al. This is an open-access article distributed under the terms of the Creative Commons Attribution License, which permits unrestricted use, distribution, and reproduction in any medium, provided the original author and source are credited.

\begin{abstract}
Ifosfamide-Related Encephalopathy (IRE) is a unique neurotoxic side effect of ifosfamide that can occur during or after its administration. The exact underlying mechanism responsible for the development of IRE is not yet known; however, mitochondrial toxicity from one or more ifosfamide metabolites has been proposed. Most cases of IRE are mild and resolve spontaneously with discontinuation of ifosfamide in addition to supportive care, but some patients progress to severe encephalopathy with the risk of seizure, coma, and even death. Electroencephalogram (EEG) abnormalities with or without apparent clinical neurotoxicity symptoms develop in a high percentage of patients treated with ifosfamide, with diffuse slowing being the most common finding; in addition, generalized periodic discharges, triphasic waves, periods of suppression, and patterns consistent with nonconvulsive status epilepticus have also been reported.

We describe a 66-year-old male with recurrent diffuse large B cell lymphoma who was admitted to the hospital for the administration of RICE (rituximab, ifosfamide, carboplatin, etoposide) chemotherapy. The patient was started on intravenous ifosfamide infusion over $24 \mathrm{~h}$. The patient became somnolent, confused, and disoriented and developed agitation $16 \mathrm{~h}$ after starting ifosfamide. The administration of ifosfamide was then discontinued, and the patient was transferred to neurocritical care for neurological monitoring. EEG showed diffuse slowing with a period of marked attenuation accompanied by intermittent rhythmic delta wave activity. The patient was given intravenous albumin and was started on methylene blue and thiamin. His mentation improved significantly and returned to baseline within the following $72 \mathrm{~h}$. The patient was eventually transferred back to the oncology floor and was discharged home in stable condition.
\end{abstract}

Keywords: Encephalopathy; Neurotoxicity; Ifosfamide; Chemotherapy; Electroencephalogram; Methylene blue

Abbreviations: IRE: Ifosfamide-Related Encephalopathy; MB: Methylene Blue; EEG: electroencephalogram; NCSE: Non Convulsive Status Epilepticus; IV: Intravenous; CT: Computed Tomography; MRI: Magnetic Resonance Imaging; CNS: Central Nervous System; CAA: Chloroacetaldehyde; EBUS: Endo Bronchial Ultrasound; NAD: Nicotinamide Adenine Dinucleotide; NADH: Nicotinamide Adenine Dinucleotide- Hydrogen; H: Hours; IVPB: Intravenous Piggyback; DLBCL: Diffuse Large B Cell Lymphoma; PICC: Peripherally Inserted Central Catheter

\section{Introduction}

Ifosfamide is an alkylating cytotoxic drug used in the treatment of several malignancies in children and adults, including lymphoma, sarcoma and ovarian and testicularcancer [1]. Among patients receiving ifosfamide, $10 \%-30 \%$ develop variable degrees of encephalopathy [2]. Clinical features of ifosfamide-related encephalopathy (IRE) are not specific, and presenting symptoms range from mild somnolence, confusion, disorientation, and sleepiness to moderate symptoms of restlessness, hallucination, agitation, involuntary limb movement, and psychosis. Severe life-threatening presentations such as seizure, coma, and death have also been reported. IRE can affect patients of all ages, including children [3-5]. Symptoms can appear insidiously, within as little as $2 \mathrm{~h}$; however, symptoms are most commonly observed 12-48 h after the initiation of intravenous (IV) ifosfamide administration. Symptoms can last up to 30 days, but usually resolve spontaneously in 1-3 days following discontinuation of ifosfamide in addition to supportive care [6]. Factors that may increase the risk of developing IRE include rapid IV infusion of ifosfamide with a short infusion time, low serum albumin levels, previous use of cisplatinum, concomitant use of aprepitant, prior history of IRE, pre-existing neurological disease, and poor functional status [7]. IRE is a diagnosis of exclusion made on a clinical basis within a broad differential diagnosis. Electroencephalogram (EEG) is a valuable diagnostic tool for evaluating patients with potential IRE [8].

Current treatment options for moderate to severe cases include Methylene Blue (MB), thiamine, and IV albumin. Moreover, 
benzodiazepines have been used in patients with Nonconvulsive Status Epilepticus (NCSE) and seizure, and dexmedetomidine (Precedex) IV infusion has been safely used for symptomatic treatment of severe agitation in patients with IRE.

\section{Case Report}

A 66-year-old male was admitted to the hospital for the administration and completion of RICE (Rituximab, Ifosfamide, Carboplatin, and Etoposide) chemotherapy for recurrent Diffuse Large B Cell Lymphoma (DLBCL). The patient had developed hypotension and hypoxia after a recent outpatient administration of Rituxan. He had a remote history of DLBCL diagnosed 19 years prior. The patient had been initially treated with six cycles of CHOP (Cyclophosphamide, Hydroxydaunorubicin, Oncovin [Vincristine], and Prednisone) chemotherapy and then experienced a relapse 2 years later. To treat the relapse, the patient received two cycles of RICE chemotherapy without significant side effects other than nausea, followed by autologous blood stem cell transplantation. The patient has remained in remission since that time but has not been followed by his oncologist for years. The patient's current presenting symptoms were hoarseness of voice, night sweats, headache, weight loss of approximately 15 pounds over 3 months, and dysphagia with odynophagia. He recently underwent an extensive work-up, including a chest Computed Tomography (CT) scan, which showed significantly enlarged mediastinal lymphadenopathy resulting in compression of the esophagus. Bronchoscopy with Endobronchial Ultrasound (EBUS)guided lymph node aspiration and endobronchial biopsy from the left main bronchus endobronchial lesions were both consistent with DLBCL. He had a recurrent malignant right pleural effusion required Pleurx catheter placement for pleural fluid drainage.

The patient's past medical history includes chemotherapy-induced and alcoholic cardiomyopathy with a recent Ejection Fraction (EF) of $40 \%$ status post placement of Automatic Implantable Cardioverter Defibrillator (AICD), a recent history of right upper extremity superficial and deep venous thrombosis involving the right subclavian, axillary, basilic, and cephalic vein, was related to placement of a PICC line, which was removed, and the patient was treated with apixaban.

Upon admission, the patient's medications included carvedilol, digoxin, losartan, spironolactone, allopurinol, apixaban, ondansetron, and promethazine. Physical examination was noteworthy for a cachectic, malnourished male with no acute distress, a weight of 65.4 $\mathrm{kg}$, a notably hoarse voice, and an alert/awake status. The patient was oriented $\mathrm{x} 3$, with no palpable cervical or supraclavicular lymph nodes and diminished breath sounds at the right base. His vital signs were a blood pressure of $80 / 49 \mathrm{mmHg}$, a pulse of 88 beats per minute, a respiration rate of 22 breaths per minute, a temperature of $97.6^{\circ} \mathrm{F}$ $\left(36.4^{\circ} \mathrm{C}\right)$, and an oxygen saturation of $92 \%$ on room air. The laboratory data are shown in table 1.

On admission, the patient was started on IV hydration with normal saline $\mathrm{NaCl} 0.9 \%$ at $50 \mathrm{~mL} / \mathrm{h}$, IV hydrocortisone $100 \mathrm{mg} \mathrm{IV} \mathrm{q} 8 \mathrm{~h}$, and proamatine $5 \mathrm{mg}$ orally three times a day. His home antihypertensive medications were held due to hypotension. On hospital day 2, the patient received aprepitant $130 \mathrm{mg}$ IV push one-time dose at 13:22 pm to prevent chemotherapy-induced nausea and vomiting followed by etoposide $180 \mathrm{mg}$ over $90 \mathrm{~min}$ at 14:19 $\mathrm{pm}$, which the patient tolerated well.

On hospital day 3 at 15:08 pm, the patient received etoposide 175 $\mathrm{mg}$ IV infusion over $90 \mathrm{~min}$ and carboplatin $465 \mathrm{mg}$ IV infusion. At $16: 30 \mathrm{pm}$, the patient was started on ifosfamide $8750 \mathrm{mg}+8750 \mathrm{mg}$ mesna (2-mercapto ethyl sulphonate) in $1000 \mathrm{~mL}$ of normal saline solution started over a 24-h IV infusion.
Table 1: Laboratory data.

\begin{tabular}{|c|c|c|}
\hline Variable & $\begin{array}{c}\text { Normal Reference } \\
\text { Range }\end{array}$ & $\begin{array}{l}\text { Admission } \\
\text { Value }\end{array}$ \\
\hline $\mathrm{Na}(\mathrm{mEq} / \mathrm{L})$ & $135-148$ & 126 \\
\hline $\mathrm{K}(\mathrm{mEq} / \mathrm{L})$ & $3.4-5.3$ & 4.6 \\
\hline $\mathrm{Cl}(\mathrm{mEq} / \mathrm{L})$ & $96-110$ & 91 \\
\hline $\mathrm{CO} 2(\mathrm{mEq} / \mathrm{L})$ & $19-32$ & 20 \\
\hline BUN (mg/dl) & $3.0-29$ & 20 \\
\hline Creatine (mg/dl) & $0.5-1.4$ & 0.9 \\
\hline Glucose (mg/dl) & $70-99$ & 87 \\
\hline Calcium (mg/dl) & $8.5-10.5$ & 11.7 \\
\hline Magnisum (mg/dl) & $1.4-2.5$ & 2.1 \\
\hline Phosphorous (mg/dl) & 2.1-4.3 & 2.6 \\
\hline Total Protien (g/dl) & $6.0-8.3$ & 4.7 \\
\hline Albumin (g/dl) & $3.5-5.2$ & 2.5 \\
\hline Alkaline phosphatase (Unit/L) & $23-144$ & 141 \\
\hline Aspartate Aminotransferase (Unit/L) & $0.0-55$ & 33 \\
\hline Alanine Aminotransferase(Unit/L) & $0.0-60$ & 21 \\
\hline Total Biliribin(mg/dl) & $0.0-1.2$ & 0.5 \\
\hline Uric acid(mg/dl) & $4.0-8.0$ & 5.6 \\
\hline $\mathrm{LDH}(\mathrm{U} / \mathrm{L})$ & $0-270$ & 764 \\
\hline White Cell Count(k/mm $\left.{ }^{3}\right)$ & $3.8-10.8$ & 8.8 \\
\hline \multicolumn{3}{|c|}{ Differential count (\%) } \\
\hline Neutrophilis & $45.0-76.0$ & 79.3 \\
\hline Lymphocytes & $14.0-51.0$ & 8.9 \\
\hline Monocytes & $0.0-14.0$ & 8.3 \\
\hline Basophils & $0.0-2.0$ & 0.9 \\
\hline Eosinophils & $0.0-7.0$ & 2.6 \\
\hline Platelet Count $\left(\mathrm{k} / \mathrm{mm}^{3}\right)$ & $130-400$ & 273 \\
\hline Heamoglobin (g/dl) & $13.8-1.2$ & 11.8 \\
\hline Hematocri (\%) & $41.0-50.0 \%$ & $35.20 \%$ \\
\hline INR & $0.9-1.1$ & 1.3 \\
\hline Prothrombin(PT)(seconds) & $11.7-13.9$ & 16.2 \\
\hline
\end{tabular}

During the early hours of infusion, there were no reported changes in mental status. At 8:36 am on hospital day 4, approximately $16 \mathrm{~h}$ after the infusion of ifosfamide + mesna was started, the patient was reported as somewhat confused and emotional according to his wife, which was attributed to possible steroid dose side effects; thus, the steroid dose was subsequently decreased.

He was noted to have mild respiratory distress, with an increased oxygen requirement of 8-10 L. The patient was given albumin $25 \mathrm{~g}$ $25 \%$ IV and one dose of furosemide $40 \mathrm{mg}$ IV while ifosfamide+mesna infusion was continued with careful monitoring. The patient's mental status deteriorated in the next few hours, and he was unable to recognize his spouse. His neurological changes appeared to be gradual in nature, and the patient became somnolent and restless, with impaired attention and periods of aphasia. He was able to briefly attend 
to voice, answered some questions appropriately, and followed some simple commands such as finger tracking but would not grip with his hand or give a thumbs-up to command, moving all extremities equally to antigravity with some resistance. His deep tendon reflexes were rated at +2 bilaterally with no ankle clonus, toes Babinski down, and no asterixis or tremor. The patient held his eyes closed when opening was attempted and withdrew from pain and deep nail bed pressure in all extremities. Coordination and gait could not be assessed due to a lack of cooperation and risk of falling. At this point, a diagnosis of toxic metabolic encephalopathy due to IRE was made, and the infusion of ifosfamide+mesna was discontinued after approximately $23 \mathrm{~h}$ of infusion, with roughly $8400 \mathrm{mg}$ of ifosfamide having been received.

The patient's laboratory data were as follows: glucose $150 \mathrm{mg} /$ $\mathrm{dL}, \mathrm{Na} 132 \mathrm{mEq} / \mathrm{L}, \mathrm{K} 2.6 \mathrm{mEq} / \mathrm{L}$ (which was immediately corrected with IV $\mathrm{KCl}$ supplement), Blood Urea Nitrogen (BUN) $23 \mathrm{mg} / \mathrm{dL}$, creatinine $0.7 \mathrm{mg} / \mathrm{dL}, \mathrm{Ca} 9 \mathrm{mg} / \mathrm{dL}, \mathrm{Mg} 1.9 \mathrm{mg} / \mathrm{dL}$, ammonia level $<10 \mu \mathrm{mol} / \mathrm{L}$ (normal reference range: 11-35), WBC 7.7, hemoglobin $11.2 \mathrm{~g} / \mathrm{dL}$, hematocrit $33 \%$, and platelet $294 \mathrm{k} / \mathrm{mm}^{3}$. Head CT showed generalized cerebral atrophy but no acute abnormality. Brain magnetic resonance imaging (MRI) was not performed due to the presence of an AICD, which is not compatible with MRI. An angio chest CT showed no pulmonary embolism but did exhibit an unchanged mediastinal lymphadenopathy, bilateral pleural effusion, and compressive atelectasis. EEG was performed using a standard protocol with EKG and a video recording showed a background of diffuse slowing with a variable frequency pattern (theta and delta wave components) and periods of incomplete suppression but no definite epileptic activity (Figures 1 and 2).

The patient was transferred to neurocritical care for neurological monitoring. He remained lethargic, confused and disoriented, mostly nonverbal (only able to verbalize a pain response), and spontaneously awake. The patient's eyes fluctuated open and closed and did not track or visually attend to the examiner or the environment.

He was started on MB $50 \mathrm{mg}$ in $50 \mathrm{~mL}$ of dextrose in water (D5W) IVPB every $6 \mathrm{~h}$ and IV thiamin $100 \mathrm{mg}$ daily. His urine turned green secondary to MB administration (Figure 3).
The patient continued to be confused, agitated, lethargic, and disoriented for the next $72 \mathrm{~h}$, requiring a bed-side sitter and four-point restraints with a Swedish belt. On day 4 of neurological critical care, his mental status improved significantly and returned to baseline. He became alert and oriented $\mathrm{x} 3$, and his respiratory distress resolved. His oxygen requirement decreased to $2 \mathrm{~L}$, and he was eventually weaned off oxygen. At this time, MB was discontinued (he had received 16 doses over 4 days) while thiamine was continued. The patient was transferred back to the oncology unit and completed his etoposide doses only; however, his hospital course was complicated by neutropenic fever, which was treated with granulocyte colonystimulating factor and broad-spectrum antibiotics, while his blood and urine culture remained negative. The patient recovered and was discharged home in stable condition.

\section{Discussion}

Metabolic encephalopathy, a potentially reversible abnormality in brain functioning, is a clinical state of global cerebral dysfunction induced by various etiologies such as hypoxia, hypercapnia, hypoglycemia, metabolic and electrolyte imbalance, medications, and toxic agents [9]. Chemotherapy-related encephalopathy due to the use of several chemotherapeutic agents, including but not limited to ifosfamide, cisplatinum, and 5-flurouracil, is a well-known condition [10]. Other forms of neurotoxicity have been reported after treatment with variable cytotoxic medications, such as aseptic meningitis, transverse myelopathy and leukoencephalopathy, peripheral neuropathy [11], and Posterior Reversible Encephalopathy Syndrome (PRES) $[12,13]$. However, IRE is a unique and serious neurotoxic side effect with a wide spectrum of insidious but nonspecific neurological presentations, ranging from sleepiness, somnolence, disorientation, impaired memory, confusion, decreased level of consciousness, agitation, restlessness, visual and auditory hallucination, personality changes, mutism, vertigo, ataxia, asterixis, [14] tremor, dysarthria, amnesia, aphasia, and muscle twitching to more severe presentations such as psychosis, [15] NCSE, [16,17] seizure, and even coma and death. Other rare presentations such as extrapyramidal involuntary movement of the limbs and face, [18] yawning, orofacial and arm

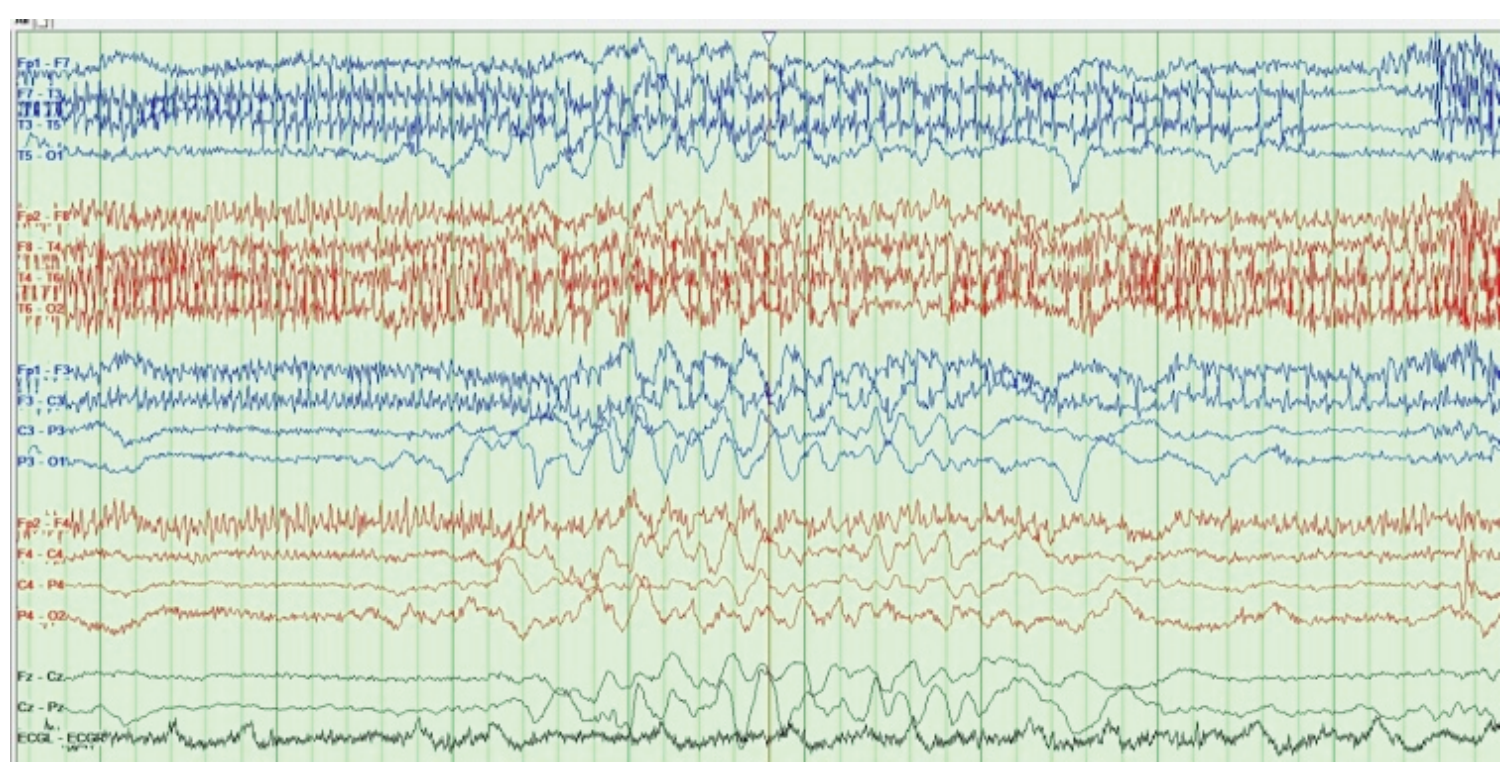

Figure 1: Suppression burst pattern.

Citation: Darwich NS, Mustafa I (2021) Encephalopathy Associated with Ifosfamide Use in the Treatment of Patient with Diffuse Large B Cell 


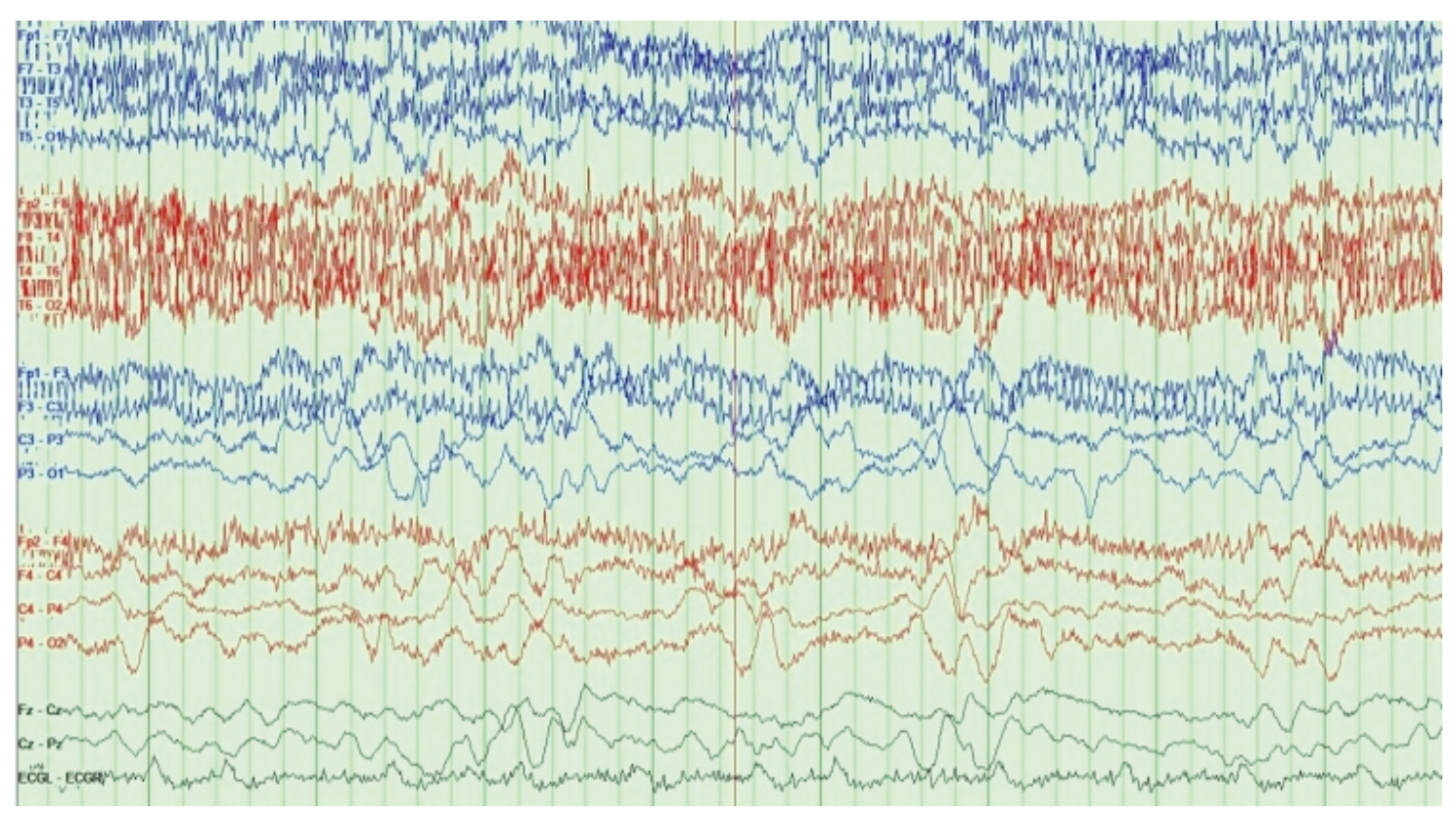

Figure 2: Delta waves.

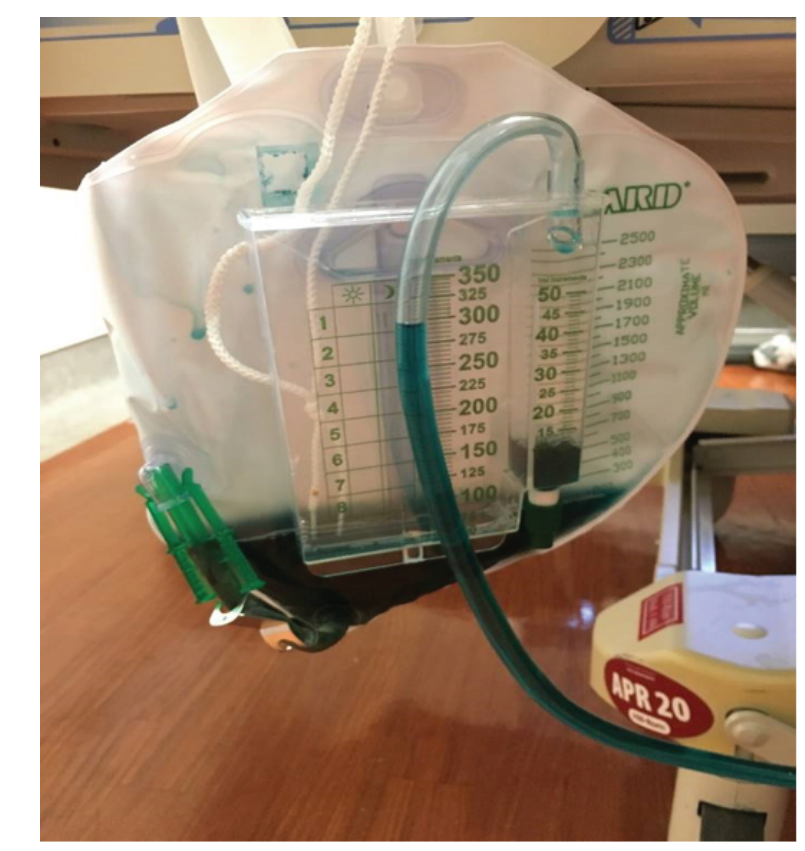

Figure 3: Green discoloration of urine after use of MB.

myoclonus, [19] catatonia, paranoia, blurred vision; delusions, hearing loss, and fecal and urinary incontinence have also been reported. Confusion is the most commonly reported symptom in patients with IRE [20].

Among patients receiving ifosfamide, $5 \%-40 \%$ develop some degree of encephalopathy; however, the true incidence rate may be higher, as mild cases can be missed and are not usually reported. The exact underlying mechanism governing the development of IRE is not yet known, but several hypotheses suggest that one or more ifosfamide metabolites may be the responsible neurotoxic compounds causing these neurological manifestations.

Ifosfamide, which is a nitrogen mustard derivative, has been widely used as a single adjuvant chemotherapeutic agent or in combination with other cytotoxic drugs to treat a variety of cancers including sarcoma, rhabdomyosarcoma, lymphoma, ovarian, and germ cell tumors such as testicular cancer. Ifosfamide is a prodrug metabolized first by cytochrome CYP-P450 and CYP3A4 in the liver to cytotoxic active metabolites, 4-hydroxy-ifosfamide and ifosfamide mustard, and other non-active metabolites such as chloroethylamine, thialysine ketamine, and Chloroacetaldehyde (CAA), which are potentially neurotoxic [21].

A proposed mechanism of ifosfamide-induced neurotoxicity suggests that these inactive metabolites may interfere with the electronbinding flavoprotein in the mitochondrial respiratory chain, resulting in a loss of energy and an accumulation of CAA, which crosses the Blood Brain Barrier (BBB), causes rapid depletion of glutathione, exerts direct neurotoxic effects, and disrupts mitochondrial respiration (Figure 4) [22].

The most common toxic side effects of ifosfamide include bone marrow suppression, hemorrhagic cystitis, nausea and vomiting, cardiac arrythmia, and neurotoxicity. Hemorrhagic cystitis, which is caused by acrolein, another ifosfamide metabolite, is usually prevented by prophylaxis-concomitant administration of mesna during ifosfamide infusion. The neurotoxic effects of ifosfamide may be mild and transient and may resolve spontaneously; however, in some cases, this condition can progress to moderate or severe encephalopathy with long-term organic brain syndrome [23] and potentially fatal complications such as coma and death [24]. Ifosfamide neurotoxicity usually manifests several hours after the initiation of infusion, within 12-48 h, although symptoms can appear as early as $2 \mathrm{~h}$ after administration. Delayed presentation of IRE has been reported, with

Citation: Darwich NS, Mustafa I (2021) Encephalopathy Associated with Ifosfamide Use in the Treatment of Patient with Diffuse Large B Cell Lymphoma. J Neurol Neurobiol 7(1): dx.doi.org/10.16966/2379-7150.174 


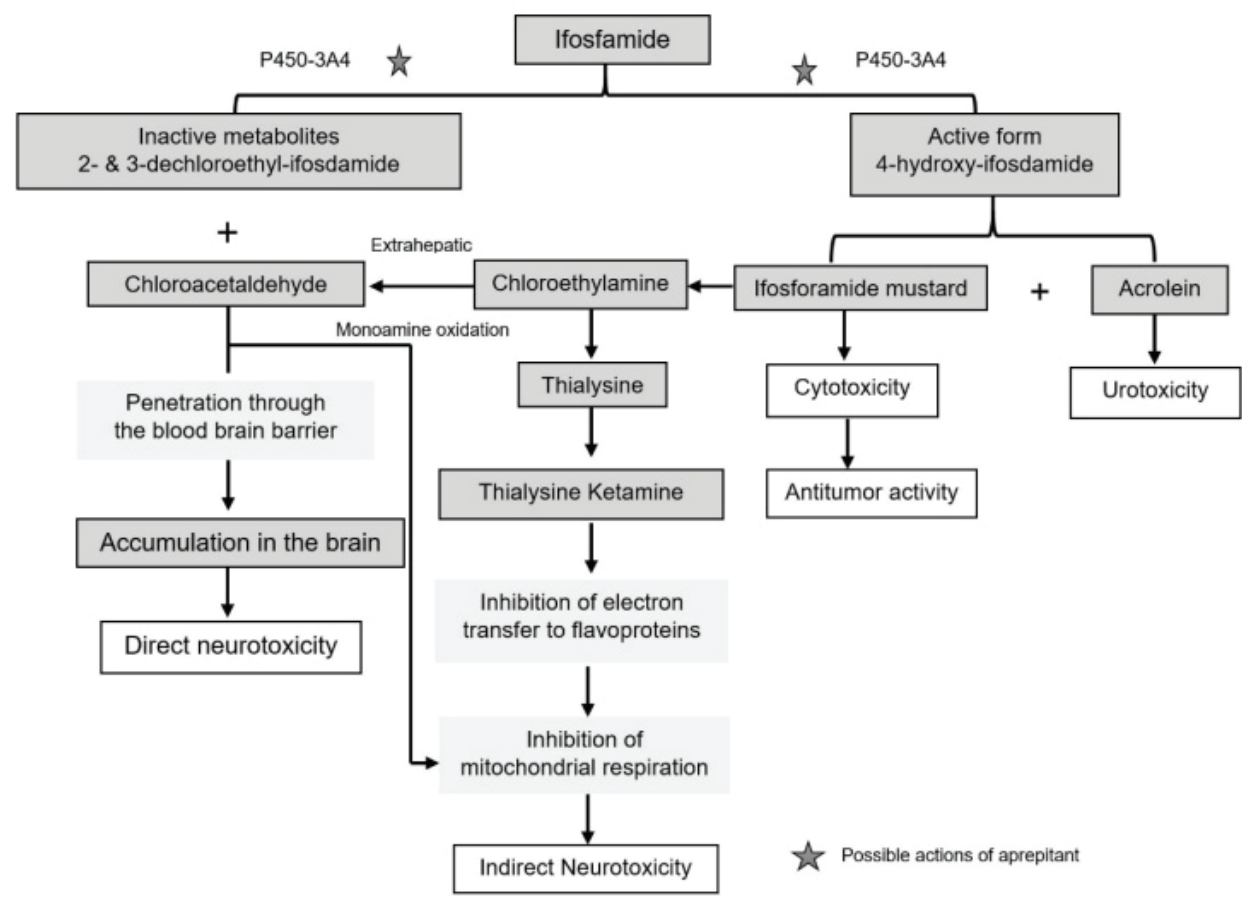

Figure 4: Metabolism of ifosfamide and proposed pathogenesis in ifosfamide-induced encephalopathy.

Reprinted with Permission from Karger Publishers Open Access. The final published version of this article is available at http://www.karger. com/?doi=10.1159/000368184.

patients developing symptoms 16 days after ifosfamide infusion [25]. Symptoms of IRE can persist for up to 30 days; however, with discontinuation of ifosfamide infusion and supportive care, symptoms usually resolve in the majority of patients within a few hours to a few days [26].

The onset, severity, duration, and recovery time of IRE symptoms vary among individual patients. The cause for this variation is unclear, but it could be due to individual variations in ifosfamide metabolism. Potential risk factors associated with IRE include low serum albumin levels, rapid IV infusion of ifosfamide with a short infusion time, previous use of cisplatinum, concomitant use of aprepitant, renal dysfunction, prior history of IRE, pre-existing neurological disorders, and poor performance status $[27,28]$.

Aprepitant, a centrally acting neurokinin-1 receptor antagonist, is used as a selective antiemetic for highly emetogenic chemotherapeutic agents [29]. The potential inhibition of CYP3A4 by aprepitant may increase the level of ifosfamide neurotoxic metabolites, resulting in the accumulation of CAA [30-32]. Concomitant administration of CPY 3A4 isoenzyme inducers such as phenytoin, phenobarbital, carbamazepine, and rifampin may increase efficacy of ifosfamide as well as its neurotoxicity.

According to the National Cancer Institute common toxicity criteria, encephalopathy is classified under neurotoxicity due to chemotherapy in four clinical grades: grade I: mild symptoms; grade II: moderate symptoms limiting instrumental Activities of Daily Life (ADL); grade III: severe symptoms limiting self-care ADL; grade IV: life-threatening consequences, with urgent intervention indicated, including seizures, toxic psychosis, or coma; grade $\mathrm{V}$ : conditions in which death occurs [33].
Most patients with IRE develop grade I or grade II neurotoxicity, while some will classify as grade III. Only approximately $10 \%-20 \%$ of patients with IRE will develop grade IV neurotoxicity.

The most common EEG findings in patients with IRE are background diffuse generalized slowing and periodic attenuation exhibited as a burst-suppression pattern with intermittent delta activity and Generalized Periodic Discharges (GPDs) with or without triphasic morphology waves; however, these findings have been observed in a variety of metabolic encephalopathy cases [34]. Occasionally, patterns consistent with NCSE, orofacial myoclonus, and epileptiform discharges, including spikes and sharp waves, have been reported, superimposed upon the pattern of background slowing. Researchers have reported a lag between EEG and clinical neurological symptoms in patients with IRE, as the EEG manifestation commonly seen in IRE can develop 12-24 h before neurotoxicity symptoms become clinically apparent and these EEG changes improve 24-48 h before clinical recovery [35].

A correlation between clinical neurotoxicity grading and EEG changes in patients with IRE has been established [36]. In grade I, EEG recordings show a reduction in the normal alpha waves (frequency: $8-13 \mathrm{~Hz}$ ) with or without small amounts of paroxysmal theta activity and a background of slowing. In grade II, predominant theta wave activity (frequency: $408 \mathrm{~Hz}$ ) with or without intermittent delta activity is seen. Grade III displays predominant delta activity with or without sharp complex waveforms, while grade IV shows continuous delta activity, complex waveforms, and triphasic waves, which represent an unrecognized subclinical status epilepticus state. A timely prolonged video EEG evaluation for patients receiving ifosfamide who develop neurological features of encephalopathy is essential for establishing a diagnosis and for identifying those patients who might have 
underlying NCSE, [37] which can present with a confusion state only and can only be diagnosed on the basis of EEG patterns demonstrating continuous generalized epileptiform activity waves with medium to high amplitude ranging from $1-1.5 \mathrm{~Hz}$ and $100-200 \mu \mathrm{V}$ spikes and wanes [38].

The diagnosis of IRE is determined on clinical grounds by ruling out other possible causes that can manifest as mental alterations in the absence of acute changes on brain imaging supported by EEG findings [39]. Conditions that mimic IRE presentation include infections with septic encephalopathy such as Central Nervous System (CNS) infection (as most of these patients with malignancies are immunosuppressed), metabolic and electrolyte disturbances, metastatic disease in the brain, hepatic encephalopathy, uremia, hypoglycemia, hypoxia or hypercapnia, hypotension or ischemic events in the brain, paraneoplastic syndrome, tumor lysis syndrome, intracranial hemorrhage, or increased intracranial pressure due to a space-occupying lesion in the brain or cerebral edema.

The cornerstone of management for patients with IRE is the immediate discontinuation of ifosfamide infusion followed by supportive care, including IV hydration, preferably with glucosecontaining fluid such as D5W. In addition, electrolyte abnormalities should be corrected, IV albumin should be administered for patients with low serum albumin, and all other drugs with potential CNS side effects, such as opioids and sedatives, should be discontinued [40]. Despite a lack of randomized prospective controlled trials proving its efficacy in IRE, MB has been commonly used in patients with moderate to severe cases of IRE, as MB can shorten the duration and severity of neurotoxic symptoms [41,42]. It has been proposed that MB may act as an electron acceptor agent and substitute for the inhibited flavoprotein. By inhibiting extrahepatic monoamine oxidase, MB may lead to a reduced formation of CAA, which in turn restores the NAD/NADH ratio and the mitochondrial respiratory chain function [43]. The use of MB in the management of IRE is supported by findings of increased glutaric acid in the urine of patients with IRE, similar to biochemical findings in patients with glutaric aciduria type II, which is a neonatal disorder of mitochondrial fatty acid oxidation, suggesting a similar pathway and response to MB treatment [44]. The recommended MB dose for IRE treatment is $50 \mathrm{mg}$ IV every 6 $h$ with continued use until the patient's neurological status returns to baseline. The co-administration of MB and a dextrose-containing IV fluid such as D5W is recommended to correct possible impairments in hepatic glucogenesis [45].

$\mathrm{MB}$ is relatively safe at this dose, and its potential benefits outweigh the risk of possible side effects. The most common side effects associated with MB use are blue-green discoloration of the urine, stool, and saliva, bladder irritation, dysuria, diarrhea, paresthesia, nausea, vomiting, abdominal and chest pain, hemolytic anemia, cardiac arrythmia, syncope, sweating, headache, dizziness, hypotension, or hypertension [46]. In rare cases, serotonin syndrome, which is a potentially lifethreatening condition, can occur, particularly in patients using serotonin enhancement drugs such as selective serotonin receptor inhibitors [47,48]. MB is contraindicated in patients with wellknown glucose 6-phosphate dehydrogenase deficiency in addition to pregnancy and lactation.

MB has also been used as a prophylaxis in patients with a known previous history of IRE when an ifosfamide challenge is needed for subsequent chemotherapy [49]. The administration of $\mathrm{MB}$ as a prophylaxis enables these patients to continue future ifosfamide chemotherapy without interruption and is known to reduce the incidence, severity, and duration of encephalopathy during ifosfamide administration; moreover, $\mathrm{MB}$ does not affect the cytotoxic effects of ifosfamide [50]. MB is usually started 6-24 h prior to ifosfamide infusion and is continued during the course of chemotherapy without interruption [51]. Oral MB can be used for prophylaxis treatment; however, its unpleasant taste and the side effects of nausea and vomiting limit its use in an oral formulation [52].

Thiamine has been used alone or in combination with MB in the treatment and prophylaxis of IRE on the basis of similarities between clinical neurological manifestations and neuropathological findings in patients with IRE and those with Wernicke encephalopathy due to severe thiamine deficiency in chronic alcoholism, suggesting a similar underlying mechanism [53]. It has been proposed that CAA, a neurotoxic metabolite of ifosfamide, interferes with the function of the active form of thiamine, Thiamine Pyrophosphorylate (TPP), by competing with TPP on its receptors at the tissue level without reducing serum thiamine levels, which may lead to failure in ATP synthesis and abnormal carbohydrate metabolism [54]; however, the exact mechanism of action of thiamine in the management of IRE remains unknown [55]. Thiamine is well tolerated and has a good safety profile with minimal side effects, including local irritation, swelling, and itching at the IV site [56].

IV albumin supplements have been used in the management of IRE patients with hypoalbuminemia, as albumin supposedly binds to ifosfamide and its neurotoxic metabolites such as CAA, blocking its passage through the Blood-Brain Barrier (BBB) and preventing direct neurotoxic effects. Meanwhile, low albumin levels increase the free fraction of available ifosfamide and its metabolites in the blood [57]. A combination of hydration, MB and Thiamine has been used prior to ifosfamide administration as a preventive regimen for IRE in patients with high risk [58].

Caution should be exercised with the use of benzodiazepines in the treatment of IRE as they can mask neurological changes and complicate the monitoring of neurological symptoms due to depressed mental function; however, benzodiazepines are still recommended for IRE patients that have developed seizures or for whom NCSE is diagnosed on EEG. Diazepam has been used with successful outcomes in these scenarios [59]. IV infusion of dexmedetomidine (Precedex), a centrally acting alpha-2 agonist, has been successfully used for the symptomatic treatment of agitation in IRE patients with good outcomes [60].

Hemodialysis should be considered in patients with severe IRE who fail to respond to discontinuation of ifosfamide administration and the use of $\mathrm{MB}$, thiamine, and albumin. Hemodialysis can reduce the concentration of both ifosfamide and its toxic metabolites by effectively removing them from the blood [61].

Most authors agree that ifosfamide infusion should be discontinued for all grades of IRE; however, for mild to moderate cases with grade I and II encephalopathy, some researchers suggest that the infusion of ifosfamide be slowed and that MB, thiamin, glucose, and albumin infusion be initiated, in addition to close monitoring of the neurological status of the patient [62].

Most cases of IRE are self-limited and resolve spontaneously with the discontinuation of ifosfamide, supportive care, and the use of MB and thiamine; however, irreversible neurological impairments in the form of cerebral atrophy, cerebellar degeneration, coma, and death have been reported.

Our patient had three potential risk factors for developing IRE, including low serum albumin levels, poor functional status, and 
concomitant use of aprepitant, even though the patient had only a onetime dose on day one of chemotherapy.

\section{Conclusion}

IRE can lead to a life-threatening condition and should thus be considered when evaluating patients with acute neurological symptoms, during or after the use of ifosfamide-based chemotherapy in patients with malignancy, as ifosfamide is becoming an important element of many chemotherapeutic regimens for treating a variety of malignancies [63]. Early recognition of IRE is the key to successful management. The exact mechanism responsible for the development of IRE is not known. Treatment of IRE is primarily focused on prompt discontinuation of ifosfamide infusion, supportive care, the exclusion of other possible underlying potential causes for encephalopathy in patients with malignancy, and timely diagnostic EEG, [64] which can confirm global cerebral dysfunction and exclude or confirm NCST, [65] as a physical neurological examination alone is not sufficiently sensitive to distinguish the diagnosis of IRE. Most cases resolve spontaneously with the discontinuation of ifosfamide infusion and supportive care alone; however, $\mathrm{MB}$, thiamin, and albumin have been used in moderate to severe cases of IRE with good outcomes [66]. Patients receiving aprepitant with ifosfamide should be carefully monitored $[67,68]$. IRE may progress to severe encephalopathy with seizure, long-term irreversible permanent structural brain dysfunction, and cerebral atrophy [69]; in addition, coma and death have been reported, even after discontinuation of infusion. Increased awareness of IRE among nursing staff and clinicians can reduce the morbidity, mortality, and length of hospital stay associated with this condition.

More randomized prospective controlled studies are needed to understand the exact mechanism responsible for developing IRE and to establish definite risk factors associated with IRE in order to prevent its occurrence and to treat IRE more effectively with scientifically proven medications guided by evidence-based medicine.

\section{Acknowledgment}

The authors thank Dana Darwich, PA-S at the Department of Physician Assistant, Kettering College, for her help with the figures and table.

\section{References}

1. Zalupski M, Baker LH (1988) Ifosfamide. J Natl Cancer Inst 80: 556566.

2. Ajithkumar T, Parkinson C, Shamshad F, Murray P (2007) Ifosfamide encephalopathy. Clin Oncol (R Coll Radiol) 19: 108-114.

3. Rieger C, Fiegl M, Tischer J, Ostermann H, Schiel X (2004) Incidence and severity of ifosfamide-induced encephalopathy. Anticancer Drugs 15: 347-350.

4. Dias E, Dias A (2019) Ifosfamide Encephalopathy in A 4yr Old with Ewing's Sarcoma. Prog Asp in Pediatric \& Neonat 2: 127-128.

5. Brunello A, Basso U, Rossi E, Stefani M, Ghiotto C, et al. (2007) Ifosfamide-related encephalopathy in elderly patients: report of five cases and review of the literature. Drugs Aging 24: 967-973.

6. Tajino T, Kikuchi S, Yamada H, Takeda A, Konno S (2010) Ifosfamide encephalopathy associated with chemotherapy for musculoskeletal sarcomas: incidence, severity, and risk factors. J Orthop Sci 15: 104111

7. David KA, Picus J (2005) Evaluating risk factors for the development of ifosfamide encephalopathy. Am J Clin Oncol 28: 277-280.
8. Gusdon AM, Malani R, Chen X (2019) Clinical and EEG Characteristics of Ifosfamide-Related Encephalopathy. J Clin Neurophysiol 36: 150154.

9. Chalela JA, Aminoff MJ, Wilterdink JL (2020) Acute toxic-metabolic encephalopathy in adult. UpToDate: 1-25.

10. Chue AL, Fernando IN, Hussain SA, Yates DA (2009) Chemotherapy related encephalopathy in a patient with Stage IV cervical carcinoma treated with cisplatin and 5-fluorouracil: a case report. Cases J 2: 8526.

11. Stone JB, DeAngelis LM (2016) Cancer-treatment-induced neurotoxicity--focus on newer treatments. Nat Rev Clin Oncol 13: 92-105.

12. Truman N, Nethercott D (2013) Posterior Reversible Encephalopathy Syndrome (PRES) after treatment with oxaliplatin and 5-fluorouracil. Clin Colorectal Cancer 12: 70-72.

13. How J, Blattner M, Fowler S, Wang-Gillam A, Schindler SE (2016) Chemotherapy-associated posterior reversible encephalopathy syndrome: a case report and review of the literature. Neurologist 21: 112-117.

14. Meyer T, Ludolph AC, Münch C (2002) Ifosfamide encephalopathy presenting with asterixis. J Neurol Sci 199: 185-188.

15. Mashhadi M, Khosravi A (2010) Neuropsychiatric Toxicity of Ifosfamide in Patients Admitted for Chemotherapy. Int J Hematol Oncol Stem Cell Res 4: 19-21.

16. Primavera A, Audenino D, Cocito L (2002) Ifosfamide encephalopathy and nonconvulsive status epilepticus. Can J Neurol Sci 29: 180-183.

17. Kilickap S, Cakar M, Onal IK, Tufan A, Akoglu H, et al. (2006) Nonconvulsive status epilepticus due to ifosfamide. Ann Pharmacother 40: 332-335.

18. Anderson NR, Tandon DS (1991) Ifosfamide extrapyramidal neurotoxicity. Cancer 68: 72-75.

19. Savica R, Rabinstein AA, Josephs KA (2011) Ifosfamide associated myoclonus-encephalopathy syndrome. J Neurol 258: 1729-1731.

20. Lee EQ, Loeffler JS, Wen PY, Savarese DMF (2020) Overview of neurologic complications of conventional non-platinum cancer chemotherapy. UpToDate.

21. Goren MP, Wright RK, Pratt CB, Pell FE (1986) Dechloroethylation of ifosfamide and neurotoxicity. Lancet 2: 1219-1220.

22. Séjournéa A, Noald S, Boonea M, Bihana C, Sassierd M, et al. (2014) Two Cases of Fatal Encephalopathy Related to Ifosfamide: An Adverse Role of Aprepitant? Case Rep Oncol 7: 669-672.

23. Zhang H, Dong J, Wang Y, Singh SK (2003) Ifosfamide-induced irreversible encephalopathy. JNMA 42: 233-234.

24. Shin YJ, Kim JY, Moon JW, You RM, Park JY, et al. (2011) Fatal Ifosfamide-induced metabolic encephalopathy in patients with recurrent epithelial ovarian cancer: report of two cases. Cancer Res Treat 43: 260-263.

25. Al-Momen A, Farhan S, Anjum FA, Aleem A (2010) Delayed ifosfamide-induced encephalopathy: A case report. J Appl Hematol 1: $113-115$.

26. Kataria PS, Kendre PP, Patel AA (2017) Ifosfamide-induced encephalopathy precipitated by aprepitant: a rarely manifested side effect of drug interaction. J Pharmacol Pharmacother 8: 38-40.

27. Lo Y, Shen LJ, Chen WH, Dong YH, Wu FL (2016) Risk factors of ifosfamide-related encephalopathy in adult patients with cancer: A retrospective analysis. J Formos Med Assoc 115: 744-751. 
28. Lee Brink A, Bowe C, Dains JE (2020) Risk Factors for IfosfamideRelated Encephalopathy in Adult Cancer Patients: An Integrative Review. J Adv Pract Oncol 11: 368-380.

29. Malhotra A, Poiesz BJ, Burgdorf AW, Gajra A (2012) Ifosfamide induced neurotoxicity secondary to concomitant aprepitant use. Adv Pharmacoepidem Drug Safety 1: 114.

30. Durand JP, Gourmel B, Mir O, Goldwasser F (2007) Antiemetic neurokinin-1 antagonist aprepitant and ifosfamide-induced encephalopathy. Ann Oncol 18: 808-809.

31. Howell JE, Szabatura AH, Seung AH, Nesbit SA (2008) Characterization of the occurrence of ifosfamide-induced neurotoxicity with concomitant aprepitant. J Oncol Pharm Pract 14: 157-162.

32. Ho H, Yuen C (2010) Aprepitant-associated ifosfamide neurotoxicity. J Oncol Pharm Pract 16: 137-138.

33. Common Terminology Criteria for Adverse Events (CTCAE) (2017) National Institutes of Health, National Cancer Institute.

34. Hartshorn JA, Foreman B (2019) Generalized periodic discharges with triphasic morphology. J Neurocrit Care 12: 1-8.

35. Meanwell CA, Blake AE, Kelly KA, Honigsberger L, Blackledge $G$ (1986) Prediction of ifosfamide/mesna associated encephalopathy. Eur J Cancer Clin Oncol 22: 815-819.

36. Feyissa A, Tummala S (2013) Clinical correlations of ifosfamide related encephalopathy with EEG: A case series. Neurology: 80.

37. Wengs WJ, Talwar D, Bernard J (1993) Ifosfamide-induced nonconvulsive status epilepticus. Arch Neurol 50: 1104-1105.

38. Kilickap S, Cakar M, Onal IK, Tufan A, Akoglu H, et al. (2006) Nonconvulsive status epilepticus due to ifosfamide. Ann Pharmacother 40: 332-335.

39. Gong QL, Fen-Ma P, Chai Y (2018) A Case of Ifosfamide Encephalopathy and its Literature Review. Med Case Rep 4: 8.

40. Meanwell CA, Blake AE, Latief TN, Blackledge G, Mould JJ, et al. (1985) Encephalopathy associated with ifosphamide/mesna therapy. Lancet 1: 406-405.

41. Donegan S (2001) Novel treatment for the management of ifosfamide neurotoxicity: Rational for the use of methylene blue. J Oncol Pharm Pract 6: 153-165.

42. Abahssain $H$, Moukafih B, Essangri $H$, Mrabti $H$, Meddah B, et al. (2021) Methylene blue and ifosfamide-induced encephalopathy: Myth or reality? J Oncol Pharm Prac1: 143-149.

43. Kupfer A, Aeschlimann C, Cerny T (1996) Methylene blue and neurotoxic mechanisms of ifosfamide encephalopathy. Eur J Clin Pharmacol 50: 249-252.

44. Sood C, O'Brien PJ (1996) 2-chloroacetaldehyde-induced cerebral glutathione depletion and neurotoxicity. $\mathrm{Br} J$ Cancer Suppl 27: S287-S293.

45. Patel PN (2006) Methylene blue for the management of Ifosfamideinduced encephalopathy. Ann Pharmacother 40: 299-303.

46. Vutskits L, Briner A, Klauser P, Gascon E, Dayer AG, et al. (2008) Adverse effects of methylene blue on the central nervous system. Anesthesiology 108: 684-692.

47. Top WM, Gillman PK, de Langen CJ, Kooy A (2014) Fatal methylene blue associated serotonin toxicity. Neth J Med 72: 179-181.

48. Snyder M, Gangadhara S, Brohl AS, Ludlow S, Nanjappa S (2017) Serotonin syndrome complicating treatment of ifosfamide neurotoxicity with methylene blue. Cancer Control 24: 1-4.
49. Turner AR, Duong CD, Good DJ (2003) Methylene blue for the treatment and prophylaxis of ifosfamide - induced encephalopathy. Clin Oncol 15: 435-439.

50. Küpfer A, Aeschlimann C, Wermuth B, Cerny T (1994) Prophylaxis and reversal of ifosfamide encephalopathy with methylene blue. Lancet 343: 763-764.

51. Gambitta L, Togliardi E, Cusmai R,C Della Costanza, S Nurro, et al. (2017) Ifosfamide induced encephalopathy: prophylaxis and therapy with methylene blue. European J Hospital Pharma 24.

52. Pelgrims J, Vos FD, Brande JV, Schrijvers D, Prove A, et al. (2000) Methylene blue in the treatment and prevention of ifosfamideinduced encephalopathy: report of 12 cases and a review of the literature. Br J Cancer 82: 291-294.

53. Hamdani M, Awan F (2006) Role of thiamine in managing ifosfamideinduced encephalopathy. J Oncol Pharm Pract 12: 237-239.

54. Buesa JM, Garcia-Teijido P, Losa R, Fra J (2003) Treatment of ifosfamide encephalopathy with intravenous thiamin. Clin Cancer Res 9: 4636-4637.

55. Imtiaz S, Muzaffar N (2010) Ifosfamide neurotoxicity in a young female with a remarkable response to thiamine. J Pak Med Assoc 60: 867-869.

56. Lin JK, Chow DS, Sheu L, Rehani B (2017) Wernicke-Like Encephalopathy Associated with Ifosfamide. Neurohospitalist 7: 4950.

57. Curtin JP, Koonings PP, Gutierrez M, Schlaerth JB, Morrow CP (1991) Ifosfamide-induced neurotoxicity. Gynecol Oncol 42: 193-196.

58. Gharaibeh EZ, Telfah M, Powers BC, Salacz ME (2019) Hydration, methylene blue, and thiamine as a prevention regimen for ifosfamide-induced encephalopathy. J Oncol Pharm Pract. 25: 17841786.

59. Simonian NA, Gilliam FG, Chiappa KH (1993) Ifosfamide causes a diazepam-sensetive encephalopathy. Neurology 43: 2700-2702.

60. Bernard PA, McCabe T, Bayliff S, Hayes D Jr (2010) Successful treatment of ifosfamide neurotoxicity with dexmedetomidine. J Oncol Pharm Pract 16: 262-265.

61. Carlson L, Goren MP, Bush DA, Griener JC, Quigley R, et al. (1998) Toxicity, pharmacokinetics, and in vitro hemodialysis clearance of ifosfamide and metabolites in an anephric pediatric patient with Wilms' tumor. Cancer Chemother Pharmacol 41: 140-146.

62. Morris E (2014) Guidelines for the use of methylene blue for the treatment and prophylaxis of ifosfamide-induced encephalitis. London Cancer: North and East. UCLH guideline.

63. Vakiti A, Pilla R, Moustafa MA, Joseph JJ, Shenoy AG (2018) Ifosfamide-Induced Metabolic encephalopathy in 2 patients with Cutaneous T-cell Lymphoma Successfully Treated with Methylene Blue. J Investig Med High Impact Case Rep 6: 1-4.

64. Feyissa AM, Tummala S (2014) Ifosfamide related encephalopathy: the need for a timely EEG evaluation. J Neurol Sci 336: 109-112.

65. Taupin D, Racela R, Friedman D (2014) Ifosfamide chemotherapy and nonconvulsive status epilepticus: case report and review of the literature. Clin EEG Neurosci 45: 222-225.

66. Kraj L, Krawczyk J, Ziarkiewicz M, Boguradzki P, Jedrzejczak WW (2018) Methylene blue in management of acute ifosfamide-induced encephalopathy. J Clin Cases Reports 1: 45-50.

67. Dixit N (2010) Ifosfamide induced neurotoxicity with addition of aprepitant. Support Care Cancer 18: S118-S119. 
68. Shimada K, Hasegawa S, Nakao S, Mukai R, Matsumoto K, et al. (2019) Adverse event profiles of ifosfamide-induced encephalopathy analyzed using the food and drug administration adverse event reporting system and the japanese adverse drug event report databases. Cancer Chemother Pharmacol 84: 1097-1105.

69. Bruggers CS, Friedman HS, Tien R, Delong R (1994) Cerebral atrophy in an infant following treatment with ifosfamide. Med Pediatr Oncol 23: 380-383. 